\title{
Age related compliance of the lamina cribrosa in human eyes
}

\author{
Julie Albon, Peter P Purslow, Wojciech S S Karwatowski, David L Easty
} P P Purslow

Department of Ophthalmology, Leicester Royal Infirmary, Leicester W S S Karwatowski

Department of Ophthalmology, Bristol Eye Hospital, Bristol

D L Easty

Correspondence to: Dr Julie Albon, Cell and Molecular Biology Unit, Department of Optometry and Vision Science,

Redwood Building, Cardiff University, King Edward VII Avenue, PO Box 905, Cardiff CF1 3XF

Accepted for publication 8 November 1999

The lamina cribrosa is a lattice-like structure, consisting of successive perforated cribriform plates across the optic nerve canal, through which pass bundles of nerve fibres. The plates are lined by basement membranes and their cores are filled with significant amounts of fibrillar collagens and elastic fibres. During ageing, the constituents of these cribriform plates are altered. ${ }^{1-4}$ It is likely that such changes will alter the mechanical behaviour of the ageing lamina cribrosa and therefore compromise its ability to support the nerve axons that pass through it.

The distribution of axonal loss in early glaucoma appears to be related to regional differences in architecture of the lamina cribrosa. The superior and inferior regions of the lamina cribrosa, which exhibit greater pore size and less dense connective tissue, are thought to be more susceptible to axonal

\begin{abstract}
Department of Optometry and Vision Science, Cardiff University, Cardiff J Albon

Department of Dairy and Food Science, The Royal Veterinary and Agricultural University, Denmark

Abstract

Aims-To investigate changes in the mechanical compliance of ex vivo human lamina cribrosa with age.

Methods-A laser scanning confocal microscope was used to image the surface of the fluorescently labelled lamina cribrosa in cadaver eyes. A method was developed to determine changes in the volume and strain of the lamina cribrosa created by increases in pressure. The ability of the lamina cribrosa to reverse its deformation on removal of pressure was also measured.

Results-Volume and strain measurements both demonstrated that the lamina cribrosa increased in stiffness with age and the level of pressure applied. The ability of the lamina cribrosa to regain its original shape and size on removal of pressure appeared to decrease with age, demonstrating an age related decrease in resilience of the lamina cribrosa.

Conclusions-The mechanical compliance of the human lamina cribrosa decreased with age. Misalignment of compliant cribriform plates in a young eye may exert a lesser stress on nerve axons, than that exerted by the rigid plates of an elderly lamina cribrosa. The resilience of the lamina cribrosa also decreased with age, suggesting an increased susceptibility to plastic flow and permanent deformation. Such changes may be of importance in the explanation of age related optic neuropathy in primary open angle glaucoma.

(Br F Ophthalmol 2000;84:318-323)
\end{abstract}

damage..$^{5}$ The arcuate retinal ganglion cell axons, which pass through these regions, are damaged first in early glaucoma.

The cause of glaucomatous axonal damage is unknown; ischaemia and mechanical damage as a result of a raised intraocular pressure or rearrangement of the cribriform plates are among the theories that have been proposed. ${ }^{7-9}$ Whatever the cause, compression of the lamina cribrosa appears to occur before detectable glaucomatous field loss. ${ }^{10}$

Age related changes in the lamina cribrosa include increases in the amounts of collagen types I, III, and IV $^{3}{ }^{4}$ within the cribriform plates, which lead to an overall increase in total collagen content. ${ }^{1-3}$ We have also demonstrated alterations in the ratio of collagen types I and III and an increase in non-enzymatic glycation. ${ }^{12}$ Remodelling of extracellular matrix within the lamina cribrosa has been attributed to the heterogeneity of astrocytes present. ${ }^{11-13}$

These changes in biochemical composition of the ageing lamina cribrosa may affect the mechanical integrity and therefore the susceptibility of elderly eyes to glaucomatous optic neuropathy. This study is the first to investigate the effect of these structural changes on the mechanical compliance of the lamina cribrosa as a function of age.

\section{Methods}

SOURCE OF TISSUE

Ten human eyes aged between 7 and 86 years were collected within 48 hours of death from the Bristol Corneal Transplant Service Eye Bank, after their corneas had been removed for transplantation. The eyes had no known pathology and diabetic eyes were excluded. Each eye was processed and analysed on the day of collection.

\section{EXPERIMENTAL DESIGN}

The posterior section of the eye, encompassing the back third of the sclera including the optic nerve, was isolated from each eye. The vitreous, retina, and choroid were removed and the interior rinsed with $0.9 \%$ saline. The glial tissue overlying the lamina cribrosa was dissected away, and the lamina cribrosa was stained using $0.5 \mathrm{mg} / \mathrm{ml}$ fluorescein isothiocyanate (Sigma) in $50 \mathrm{mM}$ bicarbonate buffer, $\mathrm{pH} 9.2$ for 1.5 hours. Following rinsing, and a 30 minute incubation in bicarbonate buffer, the tissue was then sealed to a glass slide using cyanoacrylate glue (Loctite UK, Welwyn Garden City, Herts). Air in the cavity was drawn out using a 23 gauge hypodermic needle and replaced with saline via another 23 gauge nee- 
dle connected to a graduated reservoir (Fig 1). The pressure within the cavity was controlled by raising or lowering the graduated reservoir, which was used as a simple manometer. As pressure increases the lamina cribrosa distends into a cup-like shape. The shape and volume bounded by the distorted lamina cribrosa was measured using a Bio-Rad MRC-500 scanning confocal microscope. The optical sectioning facility of this instrument permits images in the plane of the lamina cribrosa (xy scans) and in a plane perpendicular to the lamina cribrosa ( $\mathrm{xz}$ scans or yz scans) to be obtained. In each case, one $x z$ and one $y z$ scan, together with an $x y$ scan were taken in order that the three dimensional shape of the cup could be calculated. The preparation was oriented such that a line joining the two central retinal vessels was parallel to the $x$ axis of the scan. After an $x z$ and xy scan had been performed, the preparation was rotated by $90^{\circ}$, and the $\mathrm{xz}$ and xy scans were repeated.

The pressure was raised in steps from atmospheric pressure to $43 \mathrm{~mm} \mathrm{Hg}$ above atmospheric (that is, $0,11,22,33$, and $43 \mathrm{~mm}$ $\mathrm{Hg}$ ) and then lowered back down stepwise to the original pressure. At each step the lamina cribrosa was allowed to equilibrate to the new pressure level for 15 minutes before $x y$ and $x z$ scans were performed as described above. Any eye, which leaked at any stage during the performance of the experiment, was discarded and a new experiment started using a fresh eye. The mechanical compliance of the lamina cribrosa was assessed by two measures of the amount of backward bowing induced at each pressure; the volume of the distended cup formed by the lamina cribrosa and the strain in the surface of the lamina cribrosa.

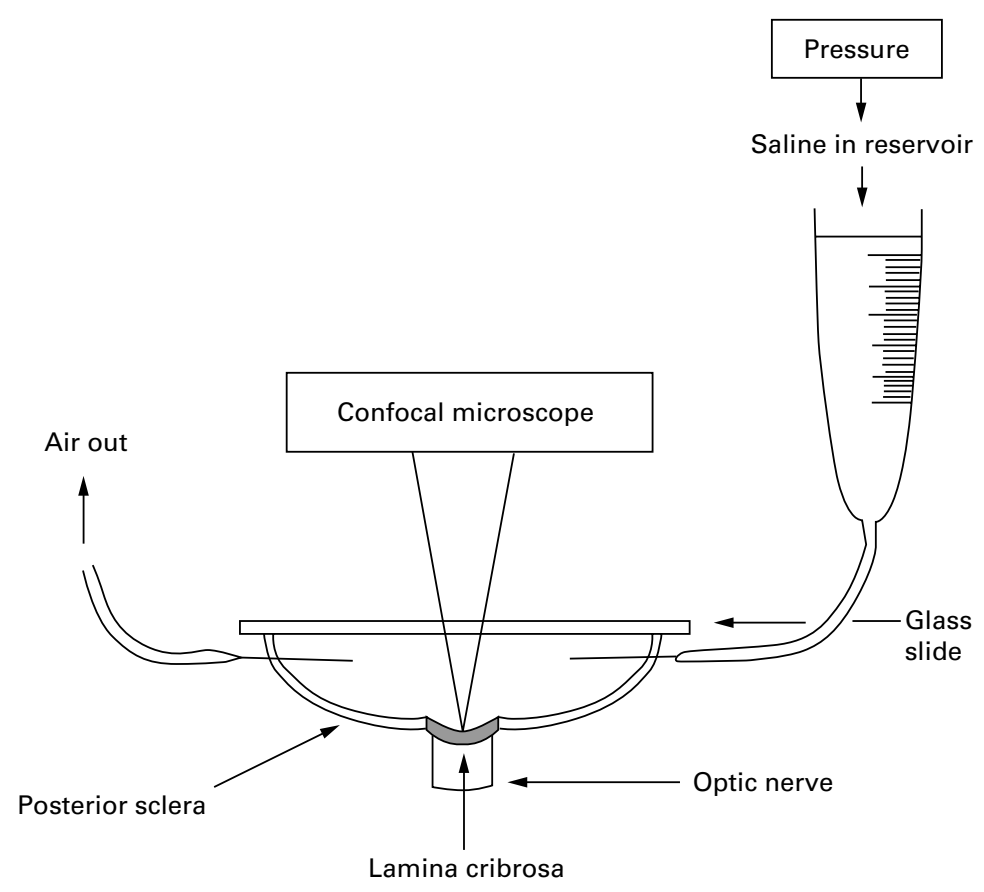

Figure 1 Schematic diagram of apparatus used to measure the change in dimensions of the lamina cribrosa on application and removal of pressure.
MEASUREMENT OF VOLUME

The photographic negatives of the $\mathrm{xz}$ scans were enlarged and the surface of the lamina cribrosa, identified by the fluorescent marker fluorescein isothiocyanate, was traced for each pressure induced deformation. The centre of gravity (c) and the area (A) bounded by each distorted cup was quantitated by a computer linked visual imaging display system (VIDS) linked to a Hipad digitiser (Houston Instrument, TX, USA).

Thus the volume (V) of the resulting cup was calculated using these parameters in the theorem of Pappus ${ }^{14}$ :

$\mathrm{V}=2 \pi \mathrm{Ac}$

The calculated volume was multiplied by 1.3346 (refractive index of saline) in consideration of the foreshortening of depth measurements produced by imaging the lamina cribrosa across an air saline interface.

CALCULATION OF REVERSIBILITY

The ability of the lamina cribrosa to recover its shape was measured by the reduction of the volume of the distended cup formed by the lamina cribrosa on removal of applied pressure.

Reversibility was calculated as: volume recovered on removal of pressure/total volume change

$\%$ Reversibility $=(100 \%-$ residual volume $) /$

where:

$$
\left(\mathrm{V}_{43}-\mathrm{V}_{0}\right)
$$

$\mathrm{V}_{43}=$ volume at $43 \mathrm{~mm} \mathrm{Hg}$ above atmospheric pressure

$\mathrm{V}_{0}=$ volume at atmospheric pressure following reduction of pressure

Residual volume $=$ (volume at atmospheric pressure following reduction of pressure) (volume at atmospheric pressure before pressure has been applied).

MEASUREMENT OF STRAIN

In order to check for anisotropy in the deformation of the lamina cribrosa, the strain in the $\mathrm{x}$ direction and the $\mathrm{y}$ direction were measured separately at each applied pressure. Each enlarged $x z$ and $y z$ scan was converted into a digital image using a Scanjet Plus (Hewlett Packard) into a computer drawing package (Deskscan). The $\mathrm{z}$ coordinates of each scan were multiplied by 1.3346 to correct for depth measurement and the $\mathrm{xz}$ and $\mathrm{yz}$ scans were reconstructed. The perimeter of the depression bound by the deformed lamina cribrosa was measured using VIDS.

The extent of the deformation of the lamina cribrosa was calculated as perimeter strain (change in length/original length at atmospheric pressure) for each pressure.

Strain $=\left(\mathrm{S}_{\mathrm{x}}-\mathrm{S}_{0}\right) / \mathrm{S}_{0}$

where $S_{0}=$ strain at atmospheric pressure before pressure increase and $\mathrm{S}_{\mathrm{x}}=$ strain induced by an applied pressure (for example, $11,22,33$, or $44 \mathrm{~mm} \mathrm{Hg}$ ) above atmospheric pressure

DETERMINATION OF MECHANICAL COMPLIANCE Differential equations of pressure-strain plots were calculated for each eye to determine the 


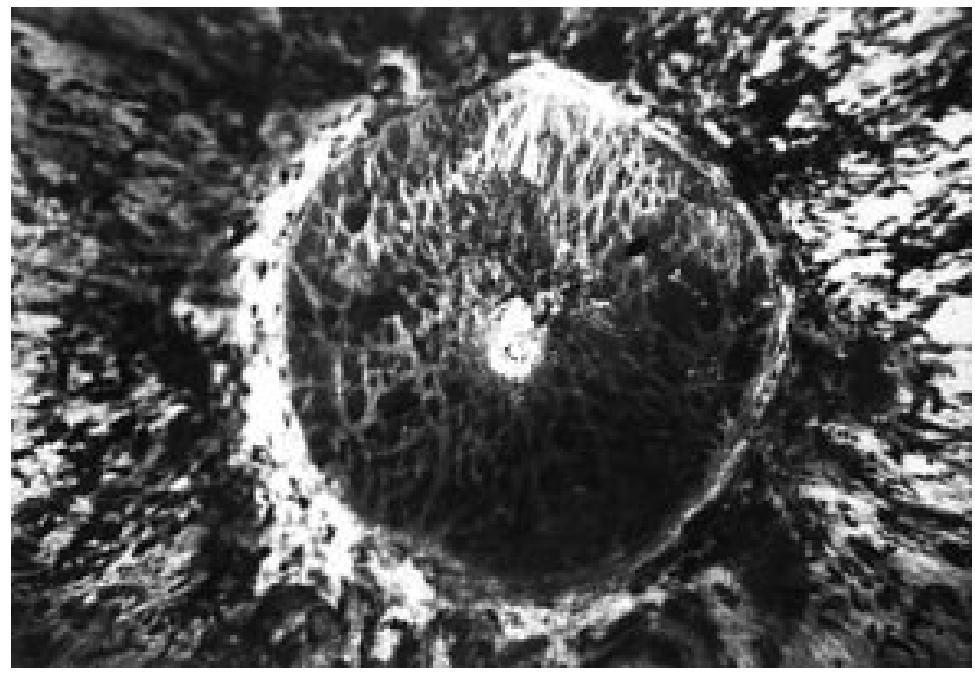

Figure 2 A typical xy scan demonstrating the fluorescently labelled anterior surface of the human lamina cribrosa, imaged using a Bio-Rad MRC-500 confocal laser scanning microscope. An average of 35 scans using Kalman were imaged for each xy scan.

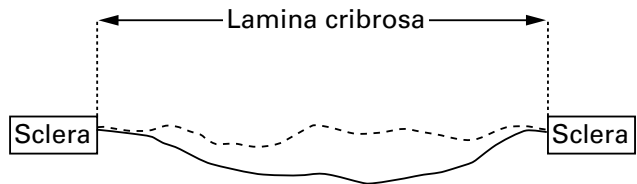

Figure 3 A confocal xz scan demonstrating the deformation of a lamina cribrosa at atmospheric pressure (solid line) and after the pressure has been increased by 43 $\mathrm{mm} \mathrm{Hg}$ (broken line). Settings included: start scan $300 \mu \mathrm{m}$ above sample surface, incremental step of 25 (2.5), motor step drive of 400

incremental gradients (change in pressure/ change in strain $=\mathrm{dP} / \mathrm{dS}$ ) of these curves at known pressure values. The incremental gradients were then plotted as a function of the pressure applied and age in order to assess the mechanical compliance of the lamina cribrosa.

STATISTICAL ANALYSIS

Spearman's rank correlation was used to test the tendency of values of $\mathrm{Y}$ (that is, reversibility, incremental gradients) to increase (or decrease) as values of $\mathrm{X}$ (age) increase.

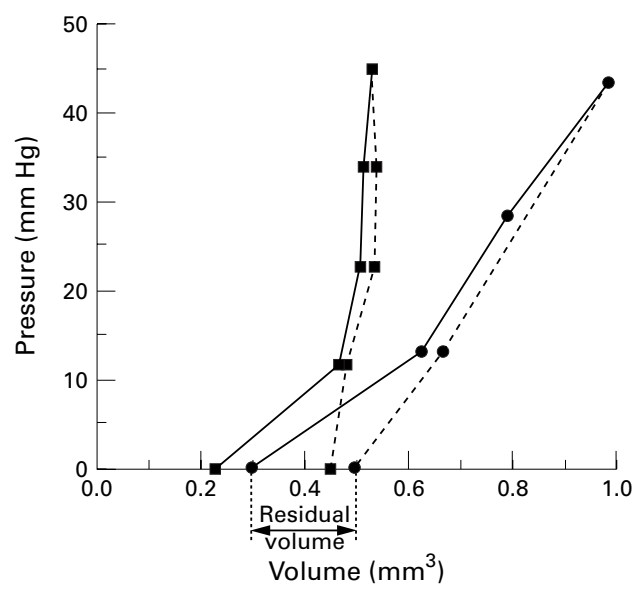

Figure 4 Pressure-volume curves of a young (9 year old: circles) and old (86 year old: squares) lamina cribrosa demonstrating their non-linearity and the difference in gradients. Solid line denotes loading curve as pressure was increased and the broken line denotes the unloading curve as pressure was decreased. Residual volume is defined as the difference between the initial volume and the volume after the removal of applied pressure.

\section{Results}

PRESSURE INDUCED DEFORMATION OF THE

LAMINA CRIBROSA

A typical xy scan is shown in Figure 2, illustrating the fluorescently labelled surface of the lamina cribrosa. Figure 3 demonstrates a typical xz scan, illustrating the backward bowing of the lamina cribrosa after the pressure had been increased to $43 \mathrm{~mm} \mathrm{Hg}$ above atmospheric pressure. These scans were used calculate the volume and strain of the deformation created at each pressure in each lamina cribrosa.

\section{Volume}

Figure 4 shows the applied pressure versus the volume bounded by the cupped lamina cribrosa for two extremes of the age range investigated. Initially a large volume change at low pressure levels was seen, but as the stress applied increased with increasing pressure, the lamina cribrosa became progressively stiffer. Stiffness is therefore defined as the ability of the lamina cribrosa to be deformed as pressure was applied.

The volume of the lamina cribrosa increased and decreased in all ages as the pressure applied was raised and lowered respectively. The gradient of the loading curve increased with age, as shown by comparison of the two curves in Figure 4. Gradients of curves from other ages fell between those demonstrated by the 9 and 86 year old in Figure 4. This illustrates that pressure induced deformations of the lamina cribrosa were greatest in the younger eyes and progressively decrease with age.

\section{Reversibility}

Reversibility of the lamina cribrosa after loading, as measured by the recovery towards original volume on reduction of pressure, decreased with age from $80 \%$ recovery in the 7 year old to less than $30 \%$ in the 86 year old (Fig 5, $r^{2}=0.776$, line shown is a fitted second order polynomial regression $\mathrm{y}=81.089$ $\left.0.89614 \mathrm{x}+4.153 \mathrm{e}^{-3} \mathrm{x}^{2}\right)$. The Spearman's rank correlation coefficient $r_{\mathrm{s}}=0.745$, which is significant at 0.013 . Therefore the ability of the ageing lamina cribrosa to recover its original

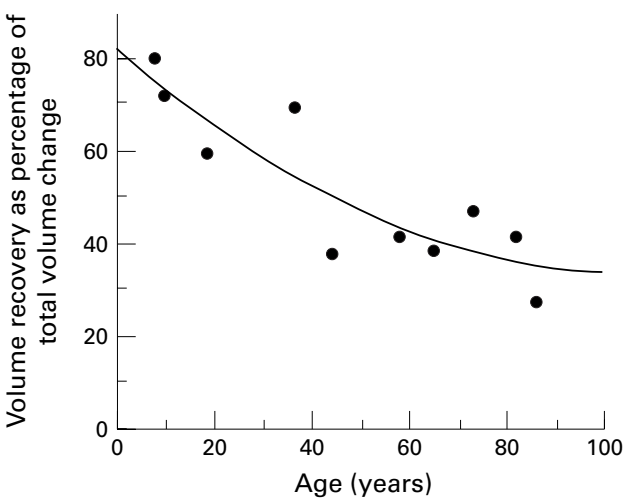

Figure 5 Reversibility of the lamina cribrosa versus age. Reversibility of the lamina cribrosa on reduction of pressure was expressed as the volume recovered as a percentage of total volume change. Line shown is a fitted second order polynomial regression $y=81.089-0.89614 x+4.153 e^{-3} x^{2}$ $r^{2}=0.776$. 

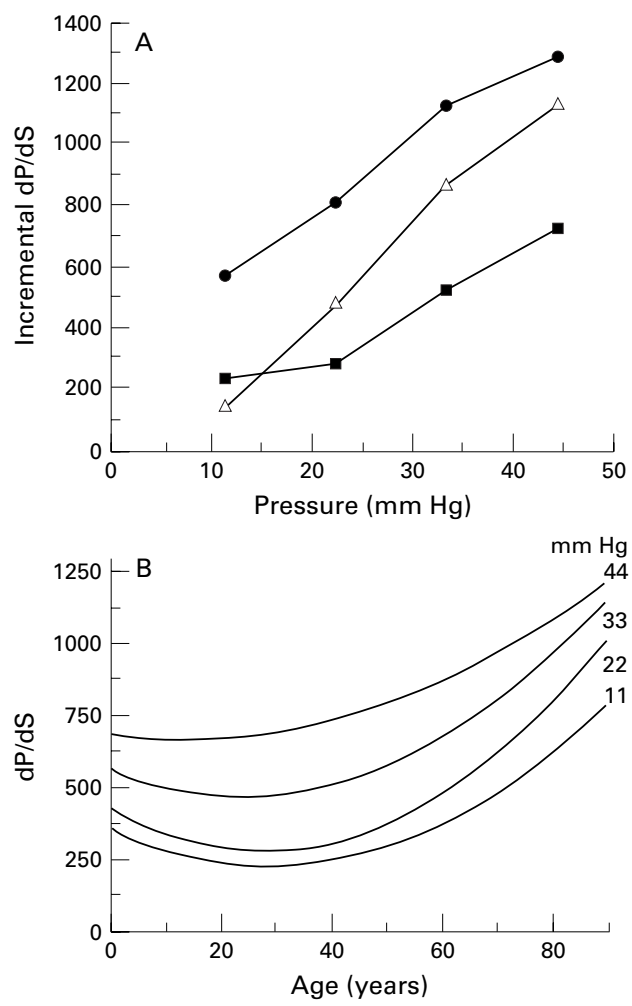

Figure 6 Incremental gradients (dP/dS, that is to say the change in pressure relative to the change in strain) were calculated for each pressure point from a polynomial

regression curve through the loading curve of each lamina cribrosa. The incremental gradient defined the changes in the mechanical compliance of the lamina cribrosa with age, as pressure was increased. (A) Incremental gradients of the pressure-strain curves for three of the 10 eyes analysed: 7 (squares), 44 (triangles) and 86 (circles) year old, versus the pressure applied to produce each strain. The incremental gradient increased with age and pressure, indicating a stiffening of the lamina cribrosa. (B) Incremental gradients $d P / d S$ versus age for given pressure increments, indicating a rapid increase in stiffness of the lamina cribrosa after 40-50 years of age. Lines show second order polynomial regression. The correlation values ( $r$ ) vary between 0.65 and 0.75 for all curves shown.

shape and volume after a pressure induced deformation was progressively reduced with age.

Strain

Pressure strain curves were similar in shape to the pressure-volume in Figure 4. Their shape illustrates the varying degrees of non-linearity in the lamina cribrosa over the range of applied stress and strain. To quantify this, gradients at several points on the pressure-strain curves were calculated from the differential of the equations from polynomial regression of these curves. The incremental gradients of each curve, plotted as a function of age and pressure (Fig 6), increased with pressure and age. Second order polynomial regression curves of incremental gradients versus age and pressure demonstrated varying correlation coefficients with $r$ values between 0.65 and 0.75 (Fig 6B). Spearman's rank correlation of this data demonstrated $r_{\mathrm{s}}$ values of $0.67,0.60,0.58$, and 0.62 which are all significant at 0.05 or above, for pressures of $11,22,33$, and $44 \mathrm{~mm} \mathrm{Hg}$ respectively. Little change in the incremental gradient was seen up to $40-50$ years of age, after which a rapid increase with age was dem-
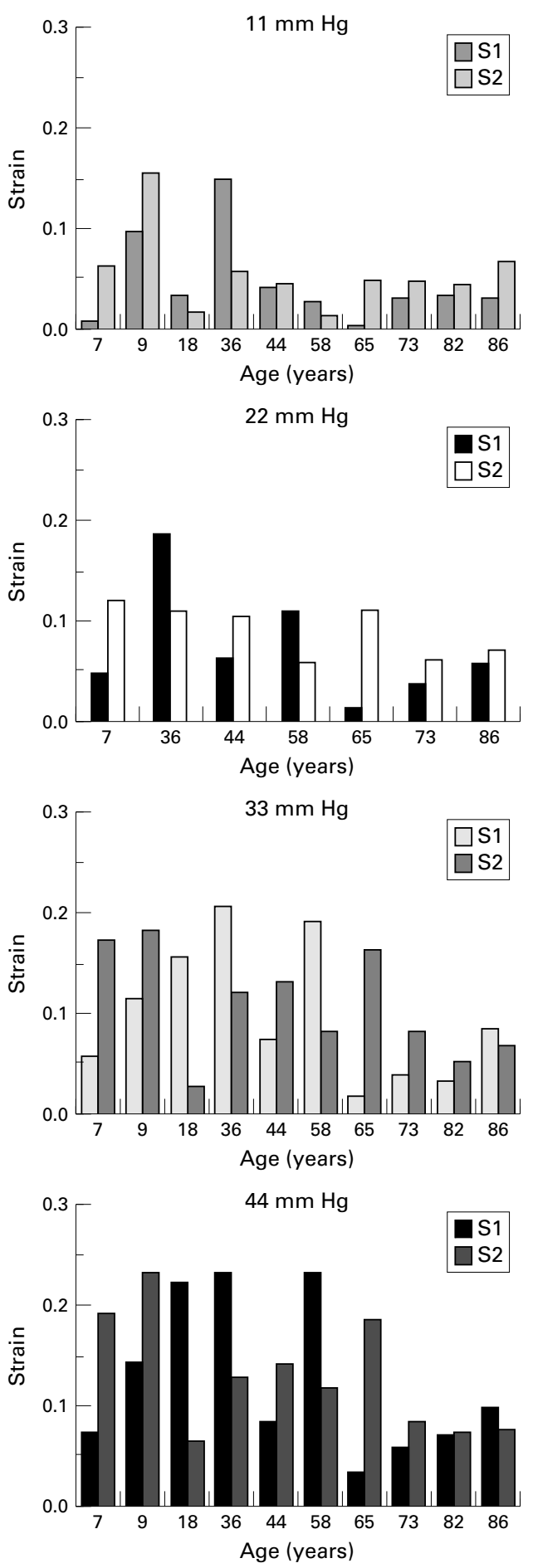

Figure 7 Strains in two directions across the lamina cribrosa S1 and S2: the strain in one of the two directions was always greater in one dimension.

onstrated (Fig 6B), implying that the mechanical compliance of the lamina cribrosa was relatively constant up to $40-50$ years of age and thereafter a rapid increase in stiffening of tissue occurred.

Some individuals demonstrated very clear differences in the strains measured in the two directions (perpendicular to each other) at all pressures, as demonstrated in Figure 7, suggesting evidence of an anisotropy in the deformation of the lamina cribrosa. 


\section{Discussion}

The mechanical properties of biological soft tissues are dependent upon the interaction of its individual components. The extracellular matrix of the lamina cribrosa is composed of elastin, collagen, and proteoglycans which define in Figure 4 the non-linear properties of a composite under stress. This mechanical behaviour can be interpreted in terms of the highly extensible elastin components and relatively stiff and inextensible collagen fibres, which have an elasticity modulus 1000 times greater than elastin. ${ }^{15}$ At low stress, it is likely that the elastin component allows the initially large volume change formed by the distending lamina cribrosa and as pressure increases and the extended collagen fibres limit further deformation, the lamina cribrosa becomes progressively stiffer.

Various in vivo techniques have been designed to quantitate optic disc topography in glaucoma patients and optic disc displacement in animal models of experimental glaucoma. ${ }^{16-22}$ Pressure induced displacement of the optic nerve head in human cadaver eyes has been monitored by scanning laser tomography, ${ }^{23}$ Doppler velocimetry, ${ }^{24}$ and radiographic evaluation of platinum wires placed in the lamina cribrosa. ${ }^{25}$ In contrast with these in vitro methods, ${ }^{23}{ }^{24}$ the technique described in this study identifies the three dimensional parameters of the pressure induced deformation at the level of the human lamina cribrosa, and uses this information to determine the biomechanical properties of the tissue as a function of age.

Previously described alterations in the extracellular matrix of the ageing human lamina cribrosa include an age related increase in the non-enzymatic collagen cross link pentosidine, total collagen and elastin content, and decreased type III to type I collagen ratio and total sulphated glycosaminoglycans content. ${ }^{12}$ Consistent with our findings in this study, such alterations may form the biochemical basis for a stiffer and less resilient structure with age.

Our results imply an overall decrease in mechanical compliance of the lamina cribrosa with age: deformations induced by a given pressure increment were greatest in the younger eyes, and decreased with age. As the lamina cribrosa ages it appears to increase in stiffness and its ability to be deformed, even under high stresses, was decreased. Simple considerations of the eye as a pressurised globe show that the stress in any part of the ocular shell, including the lamina cribrosa, is related to both intraocular pressure and the radius of the structure; $\sigma=\mathrm{PR} / 2 \mathrm{t}$, where $\sigma=$ circumferential stress, $\mathrm{P}=$ intraocular pressure, $\mathrm{R}=$ radius of curvature at that part of the globe, and $t=$ thickness. ${ }^{26}$ Thus in large eyes or when the lamina cribrosa is cupped (that is, in glaucoma or the elderly ${ }^{27}$ ), the effective radius of the entire pressure vessel is greater at this point, and therefore the stress is greater. In support of this, finite element modelling of the optic nerve head suggests that IOP related stress is concentrated at the site of early glaucomatous axonal damage. ${ }^{28}$
Reversibility of the lamina cribrosa towards original volume on reduction of pressure decreased with age, indicative of decreased resilience. When force is applied and then removed cyclically, many materials (including biomaterials) demonstrate fully recoverable (that is, elastic) deformations up to a certain point (the elastic limit). However, after the elastic limit, plastic flow occurs and the internal structure of the tissue may be permanently altered. In such cases strain does not return to zero on removal of stress. Plastic flow can continue in a tissue until it reaches its failure strength. In this study, the difference between the volume after complete removal of pressure and the original volume is known as the residual volume (see Fig 4) and is representative of permanent deformation (plastic flow) which has occurred within the lamina cribrosa. The age related decrease in reversibility following pressure induced deformation implies that permanent deformation in structure as a result of plastic flow increases with age. In vivo studies in monkeys have demonstrated chronic posterior deformation of the optic disc surface, indicative of plastic flow, 3-4 weeks following the onset of experimentally induced glaucoma. ${ }^{19}$

Consistent with our findings of higher resilience in younger laminae cribrosae is the reversibility of cupping seen in infant glaucoma ${ }^{29} 30$ and young adults, ${ }^{31}{ }^{32}$ perhaps due to higher yield points or their ability to undergo larger deformations up to their elastic limit. The degree of damage and mechanical compliance of the cribriform plates differs depending on age and the level of pressure applied; it is likely that age related stiffening of the lamina cribrosa limits its ability to distend before permanent deformation occurs.

These alterations in mechanical properties of the ageing lamina cribrosa are likely to influence the susceptibility of the retinal ganglion cell axons to damage due to the close anatomical arrangement of the cribriform plates and the nerve axons, which pass through them. In a young eye the stress exerted on the nerve axons during the compression and rearrangement of the cribriform plates may be effectively much less than in the more rigid structure of the elderly lamina cribrosa. The change in mechanical compliance appears to be most marked after 40-50 years, the age at which the incidence of primary open angle glaucoma (POAG) is increased.

In primary open angle glaucoma, the intraocular pressure is often raised and larger fluctuations in the diurnal variation of intraocular pressure occur. Each time the pressure fluctuates above the yield point, the extracellular matrix of the lamina cribrosa may undergo plastic flow with resultant permanent damage. Rigidity of the lamina cribrosa increases with progression of the disease, ${ }^{25}$ perhaps indicative of accumulative damage within the connective tissue plates, compatible with total misalignment of the cribriform plates seen in advanced glaucoma. ${ }^{10}$ The alteration in mechanical compliance of the lamina cribrosa may be significant in the 
pathogenesis of glaucomatous optic neuropathy. Assessing and understanding the changes both regionally and globally within the lamina cribrosa may be useful as a predictive factor for the development of POAG. ${ }^{25}$

The authors would like to thank the CTS Eye Bank, Bristol Eye Hospital for the provision of eyes and Professor Allen Bailey for allowing this work to be carried out within the Muscle and Collagen Research Group in the Department of Clinical Veterinary lagen Research Group in the Department of Clinical Veterinary
Science at the University of Bristol, Langford. This project was Science at the University of Bristol, Langford. This project was
supported by the Guide Dogs for the Blind Association, National Eye Research Council, and the Iris Fund.

1 Albon J, Karwatowski WSS, Avery N, et al. Changes in the collagenous matrix of the ageing human lamina cribrosa. Br f Ophthalmol 1995;79:368-75.

2 Albon J. An investigation into the age-related changes in the Albon J. An investigation into the age-related changes in the extracellular matrix of the human lamin
thesis. Bristol: University of Bristol, 1995.

3 Hernandez MR, Luo XX, Andrzejewska W, et al. Agerelated changes in the extracellular matrix of the human optic nerve head. Am 7 Opthhalmol 1989;107:476-84.

4 Morrison JC, Jerdan JA, Dorman ME, et al. Structural proteins of the neonatal and adult lamina cribrosa. Arch Ophthalmol 1989;107:1220-4.

5 Quigley HA, Addicks EM. Regional differences in the structure of the lamina cribrosa and their relation to glaucomatous optic nerve damage. Arch Ophthalmol 1981;99:13743.

6 Radius RL, Gonzales M. Anatomy of the lamina cribrosa in human eyes. Arch Ophthalmol 1981;99:2159-62.

7 Emery JM, Landis D, Paton D, et al. The lamina cribrosa in normal and glaucomatous human eyes. Trans Am Acad Ophthalmol Otolaryngol 1981;78:OP290-7.

8 Quigley HA, Flower RW. The mechanism of acute intraocular pressure-induced optic nerve damage. Invest Ophthalmol lar pressure-induced

9 Tengroth B, Ammitzboll T. Changes in the content and composition of collagen in the glaucomatous eye-basis of a new hypothesis for the genesis of primary open-angle glaucoma. Acta Ophthalmol 1984;62:999-1008.

10 Quigley HA, Hohman RM, Addicks EM, et al. Morphological changes in the lamina cribrosa correlated with neural loss in open-angle glaucoma. Am f Ophthalmol 1983;95 673-91.

11 Kobashyi S, Vidal I, Pena JD, et al. Expression of neural cell adhesion molecule (NCAM) characterises a subpopulation of type I astrocytes in human optic nerve head. Glial 1998, 20:262-73.

12 Hernandez MR, Igoe F, Neufeld AH. Cell culture of the human lamina cribrosa. Invest Ophthalmol Vis Sci 1998;29: 78-89.

13 Hernandez MR, Hanley NM, Neufeld AH. Localisation of collagen types I and IV mRNAs in human optic nerve head by in situ hy $2169-77$.
14 Burrington RS. Handbook of mathematical tables and formulae 4th ed. New York, London: McGraw-Hill, 1965. 5 Fung YC. Biomechanics. Berlin: Springer-Verlag, 1981.

16 Azuara-Blanco A, Spaeth GL. Methods to objectify reversibility of glaucomatous cupping. Curr Opin Ophthalmol 1997;8:50-4.

17 Zeimer RC. Noninvasive measurement of optic nerve-head compliance by laser Doppler velocimetry. F Opt Soc Am 1981;71:499-502.

18 Burgoyne CF, Quigley HA, Thompson HW, et al. Measurement of optic disc compliance by digitised image analysis in the normal monkey eye. Ophthalmology 1995;102:1790-9.

19 Burgoyne CF, Varma R, Quigley HA, et al. Early changes in optic disc compliance and surface position in experimental glaucoma. Ophthalmology 1995;102:1800-9.

20 Burgoyne CF, Varma R, Quigley HA, et al. Global and regional detection of induced optic disc change by digitised image analysis. Arch Ophthalmol 1994;112:1261-8.

21 Coleman AL, Quigley HA, Vitale S, et al. Displacement of the optic nerve head by acute changes in intraocular pressure in monkey eyes. Ophthalmology 1991;98:35-40.

22 Zeimer RC, Slocum J, Wilensky JT, et al. Noninvasive measurement of optic nerve head mechanical compliance in normal and ocular hypertensive beagles. Curr Eye Res in normal and

23 Yan DB, Flanagan JG, Farra T, et al. Study of regional deformation of the optic nerve head using scanning laser tomography. Curr Eye Res 1998;17:903-16.

24 Levy NS, Crapps, EE. Displacement of optic nerve head in response to short-term intraocular pressure elevation in human eyes. Arch Ophthalmol 1984;102:782-6.

25 Zeimer RC, Ogura Y. The relation between glaucomatous damage and optic nerve head mechanical compliance. Arch Ophthalmol 1989;107:1232-4.

26 Purslow PP, Karwatowski WSS. Ocular elasticity. Is engineering stiffness a more useful characterisation parameter than ocular rigidity? Ophthalmology 1996;168:168692.

27 Garway-Heath DF, Wolstein G, Hitchings RA. Aging changes of the optic nerve head in relation to open-angle glaucoma. Br F Ophthalmol 1997;8:840-5.

28 Bellezza AJ, Hart RT, Martinez ML, et al. IOP-related stress within the load-bearing connective tissues of the scleral canal wall is substantial at even low levels of IOP by preliminary finite element modelling. Invest Ophthalmol Vis Sci 1998;39:S27.

29 Schaffer RN, Hetherington J. The glaucomatous disc in infants:a suggested hypothesis for disc cupping. Trans $\mathrm{Am}$ Acad Ophthalmol Otolaryngol 1969;73:929-35.

30 Quigley HA. Childhood glaucoma: results with trabeculotomy and study of reversible disc cupping. Ophthalmology 1992;89:219-26.

31 Robin AL, Quigley HA, Pollack IP, et al. An analysis of visual acuity, visual fields, and disk cupping in childhood glaucoma. Am f Ophthalmol 1979;88:847.

32 Rath EZ, Shin DH, Kim C, et al. Relationship between optic disc cupping change and intraocular pressure control in adult glaucoma patients. Graefes Arch Clin Exp Ophthalmol adult glaucoma patie 